\title{
ORIGINAL ARTICLE Multilocus estimation of selfing and its heritability
}

\author{
NS McClure ${ }^{1}$ and MC Whitlock ${ }^{2}$
}

We describe a new method of estimating the selfing rate $(S)$ in a mixed mating population based on a population structure approach that accounts for possible intergenerational correlation in selfing rate, giving rise to an estimate of the upper limit for heritability of selfing rate $\left(h^{2}\right)$. A correlation between generations in selfing rate is shown to affect one- and two-locus probabilities of identity by descent. Conventional estimates of selfing rate based on a population structure approach are positively biased by intergenerational correlation in selfing. Multilocus genotypes of individuals are used to give maximumlikelihood estimates of $S$ and $h^{2}$ in the presence of scoring artifacts. Our multilocus estimation of selfing rate and its heritability (MESH) method was tested with simulated data for a range of conditions. Selfing rate estimates from MESH have low bias and root mean squared error, whereas estimates of the heritability of selfing rate have more uncertainty. Increasing the number of individuals in a sample helps to reduce bias and root mean squared error more than increasing the number of loci of sampled individuals. Improved estimates of selfing rate, as well as estimates of its heritability, can be obtained with this method, although a large number of loci and individuals are needed to achieve best results.

Heredity (2012) 109, 173-179; doi:10.1038/hdy.2012.27; published online 23 May 2012

Keywords: selfing; mixed mating system; descent measures; heritability; bias of selfing rate estimates

\section{INTRODUCTION}

Hermaphroditic organisms can reproduce by means of three strategies: outcrossing, bi-parental inbreeding and selfing. Outcrossing involves cross-fertilization with a randomly selected mate and is achieved by either the export or import of gametes. Bi-parental inbreeding is a form of nonrandom mating with a closely related relative, whereas selfing is an extreme form of inbreeding, which results in genes of each offspring coming from a single parent. The occurrence of one of these strategies in the mating system of a population does not necessarily exclude other strategies from occurring concurrently; however, the dominance of a single strategy in a population can imply that specific factors exist to promote or inhibit other strategies. For example, limited pollinators, repeated bottleneck or colonization events by few individuals, and selection for locally adaptive traits promote selfing, whereas selfing is inhibited by selfincompatibility, dichogamy, heterostyly and temporal and spatial environmental variation (Lande and Schemske, 1985; Barrett, 2002; Goodwillie et al., 2005; Avise, 2011). These reproductive strategies are influenced by many ecological and evolutionary factors, which determine the success and proportions of selfed, outcrossed and inbred offspring in a population.

Mating systems exist upon a continuum of selfing rates limited by the two extremes of complete selfing and complete outcrossing (Lande and Schemske, 1985; Goodwillie et al., 2005). There are populations that mostly outcross with low selfing rates in the $0-0.2$ range, and there are populations that predominantly self with rates from 0.8 to 1 . Customarily, populations with selfing rates between 0.2 and 0.8 are defined as intermediate selfing populations. Although hermaphroditic plants (especially flowering plants) are the most commonly studied organisms for mating systems, the selfing rates of hermaphroditic animals, such as mollusks and trematodes, sometimes show characteristics of mixed mating (Jarne and Auld, 2006; Escobar et al., 2011). However, it is also realized that selfing rate estimates can be adversely affected by scoring artifacts if not accounted for (David et al., 2007; Escobar et al., 2011). The occurrence of these selfing rates in nature has provoked much theoretical research into evolutionary dynamics of selfing (Barrett, 2002; Goodwillie et al., 2005; Charlesworth and Willis, 2009; Avise 2011).

There are two standard methods used to estimate the selfing rate of a population; these are known as the progeny array (Ritland and Jain, 1981) and the population structure approaches (Fyfe and Bailey, 1951; see also Jarne and David, 2008). The progeny array approach estimates selfing rate from comparing mother and offspring genotypes at multiple loci (Ritland and Jain, 1981; Jarne and David, 2008). Although the population structure approach is traditionally based on the relationship between the selfing rate and the inbreeding coefficient of a population at equilibrium, it has recently been improved to incorporate data from multiple loci and account for genotyping errors (David et al., 2007; Jarne and David, 2008).

Here, we propose a new method of estimating selfing rate from multilocus genotypes as well as providing an estimate of the upper limit for the heritability of selfing rate. The novelty of this approach stems from estimating the correlation in selfing rate across generations. An intergenerational correlation in selfing rate arises from a higher propensity to self by individuals in selfing lineages, which could occur as a result of shared genetic or environmental factors between parent and offspring. In either case,

${ }^{1}$ Department of Biology, Queen's University, Kingston, Ontario, Canada and 2Department of Zoology, University of British Columbia, Vancouver, British Columbia, Canada Correspondence: NS McClure, Department of Biology, Queen's University, Kingston, Ontario, Canada K7L 3N6.

E-mail: nathan.mcclure@queensu.ca

or MC Whitlock, Department of Zoology, University of British Columbia, Vancouver, British Columbia, Canada V6T 1 Z4.

E-mail:whitlock@zoology.ubc.ca

Received 8 November 2011; revised 23 March 2012; accepted 2 April 2012; published online 23 May 2012 
the propensity to self is inherited. We can use regression in offspring selfing rate on parent selfing rate as another way of quantifying the intergenerational correlation in selfing rate. An estimate of the slope of the regression in selfing rates is, by definition, related to a measure of heritability of selfing rate. However, unlike a strict estimate of narrow-sense heritability, the heritability we have defined here may include correlations between parents and offspring caused by shared environmental factors that happen to exist in nature (in addition to genetic factors). Therefore, an estimate of heritability produced in this manner will likely be an estimate of its upper limit.

The method of estimating selfing rate and its heritability uses probabilities of identity from multiple loci simultaneously. Accounting for the correlation between generations in selfing rate helps reduce the bias of selfing rate estimates based on the population structure method. If there is a correlation in selfing rate between generations, then some lineages within a population will have high homozygosity at multiple loci and other lineages will have levels of heterozygosity at all loci as expected from random mating. The correlation in selfing rate between generations affects the probability that multiple loci in an individual are identical by descent. Thus, if there is heritability in the propensity to self, then measures of the pattern of homozygosity at multiple loci allow for the potential to estimate heritability of selfing rate. In the extreme case, a population consisting of a mixture of purely selfing and purely outcrossing lineages (and therefore a high heritability of selfing) would have an equilibrium probability of identity by descent equal to the population's selfing rate $(S)$, not $S /(2-S)$ as predicted by the classical result for a single-locus inbreeding coefficient. We derive a novel methodology based on likelihood to perform this estimation.

\section{MATERIALS AND METHODS}

\section{Selfing and the probability of identity by descent}

We will first define some terms that will be used to derive an estimate of the heritability of selfing rate while revealing that the probability of identity of alleles chosen from the same individual is affected by the correlation between generations in selfing rate. The inbreeding coefficient, $F$, is the probability that two alleles in a diploid individual are identical by descent. We will also use another term to describe the probability that two alleles at one locus within a diploid individual are identical by descent while at the same time two alleles at another locus are also identical by descent. This is a so-called two-locus descent measure and will be referred to here as $\psi$ (Cockerham and Weir, 1973).

The probability that the parent of an individual who selfs also selfed will be designated as $\rho$, while the selfing rate, $S$, is the proportion of individuals who have only a single parent. $S$ and $\rho$ can predict the distribution of selfing history in lineages. The complement of $\rho$ is the event that an individual who selfs has parents that outcrossed. The parameter, $\rho$, is assumed to be independent from generation to generation such that the probability of a particular number of generations of selfing $(x)$ in a lineage since the last ancestor that outcrossed is $\varphi(x, S, \rho)=S(1-\rho) \rho^{x-1}$ (for $x \geqslant 1$ ). We will use the notation $\varphi(x, S, \rho)$ to represent the probability that there have been exactly $x$ ancestral selfing generations in a lineage since the last ancestor that outcrossed. For 0 generations of selfing $(x=0), \varphi(x, S, \rho)=(1-S)$. Furthermore, the one- and two-locus probabilities of identity by descent for a lineage with $x$ generations of selfing history are:

$$
F(x)=1-\left(\frac{1}{2}\right)^{x}
$$

and

$$
\psi(x+1)=\frac{(1+2 F(x)+\psi(x))}{4},
$$

where $\psi(0)=0$. Using Equations 1 and 2, with some algebra we find:

$$
\begin{aligned}
\psi(x) & =\sum_{y=1}^{x}\left(\frac{1}{4}\right)^{y}+\sum_{y=1}^{x-1}\left(\frac{1}{4}\right)^{x-y} 2\left(1-\left(\frac{1}{2}\right)^{y}\right) \\
& =1-2^{1-x}+4^{-x}=(F(x))^{2} .
\end{aligned}
$$

To determine the equilibrium values of the descent measures we must account for all possible selfing lineages within a mixed mating population and average their effects over the probability that an individual with $x$ generations of selfing history is identical by descent at one or two loci. A generation of outcrossing immediately returns $F$ in the lineage to 0 . Thus, the single-locus probability of identity by descent at equilibrium is the weighted average:

$$
F=\sum_{x=1}^{\infty} S(1-\rho) \rho^{x-1}\left(1-\left(\frac{1}{2}\right)^{x}\right) .
$$

In reality, given that a large number of generations of descendants self, the probability that the ancestor also selfed is somewhat greater than in a more recent ancestor with fewer subsequent generations of selfing. This is because with non-genetic reasons for selfing mixed with genetic, the greater the number of descendant generations of selfers the more likely it is that the lineage continues to self for genetic reasons. However, Equation 4 is a reasonable approximation of the probability of a history of selfing, particularly when $\rho$ is not very close to 1 (so that the sum is dominated by the effects of recent generations).

Note also that it is impossible, by Equation 4, to differentiate between correlation in selfing over generations (as assumed in Equation 4) and a change in selfing rate before the last generation. In either case, the ancestral selfing rates would be higher for selfing lineages, and in either case there is no signal of past selfing in outcrossed individuals. The equations we present here for estimating heritability therefore require the assumption that selfing rate is not changing rapidly over time, an assumption shared by all methods that estimate selfing rates through inbreeding coefficients.

Equation 4 reduces (by way of the binomial series) to

$$
F=\frac{S}{2-\rho} \text {. }
$$

Similarly, the equilibrium two-locus probability of identity by descent is:

$$
\begin{aligned}
\psi=\sum_{x=1}^{\infty} S(1-\rho) \rho^{x-1} \psi_{x} & =\sum_{x=1}^{\infty} S(1-\rho) \rho^{x-1}\left(1-2^{1-x}+4^{-x}\right) \\
& =\frac{S(2+\rho)}{(\rho-4)(\rho-2)} .
\end{aligned}
$$

Equations 5 and 6 explicitly show that one- and two-locus measures of identity by descent are affected by the correlation between generations in selfing rate $(\rho)$. For instance, if a population consisted of a mixture of lineages that always selfed and lineages that always outcrossed $(\rho=1), F$ would equal $\psi$ and $S$ would equal $F$. Similarly, for a population with an intermediate $\rho, F$ and $\psi$ would still be affected by the correlation between generations in selfing rate rather than being solely governed by $S$.

We can now derive an estimate of the heritability of selfing rate $\left(h^{2}\right)$ as a function of $S$ and $\rho$. The heritability of selfing rate is by definition the covariance of parental selfing rate and offspring selfing rate divided by the variance of selfing rate among parents. As such, this heritability includes the effect of additive genetic variance, but it also includes correlations between parents and offspring caused by common environmental effects shared by parent and offspring. The covariance of the parent and offspring generations in their propensity to self is:

$$
\operatorname{COV}\left(S_{P}, S_{O}\right)=E\left[\left(S_{P}-E\left(S_{P}\right)\right)\left(S_{O}-E\left(S_{O}\right)\right)\right]
$$

where $E\left(S_{P}\right)$ and $E\left(S_{O}\right)$ are both equal to $S$, the average selfing rate, according to the assumption that $S$ is constant. Therefore, the equation simplifies to

$$
\operatorname{COV}\left(S_{P}, S_{O}\right)=E\left(S_{P} S_{O}\right)-S^{2} .
$$

Under a Bernoulli model of selfing, where 1 indicates the event in which parents and offspring self and 0 otherwise, $E\left(S_{P} S_{O}\right)$ is equal to $\rho S$, which means that $\operatorname{COV}\left(S_{B} S_{O}\right)=S(\rho-S)$. By a similar method, the variance of selfing 
rate among parents is found to be $S(1-S)$. Therefore, heritability of selfing rate is

$$
h^{2}=\frac{\rho-S}{1-S} .
$$

From Equation 7 it is clear that when $\rho=S$, or in other words when the tendency of offspring to self is not correlated with whether their parents selfed, the heritability of selfing rate is zero. On the other hand, when selfing offspring always have selfing parents $(\rho=1)$, the heritability of selfing rate is 1 . It is important to remember that this 'heritability' reflects all sources of correlation between parent and offspring, including the possibility of shared environmental effects. If the environmental correlation between parents and offspring is considered likely to be zero or positive, then $h^{2}$ as measured here will represent an upper bound to the narrow-sense heritability.

Equation 5 shows that the one- and two-locus probability of identity by descent measures are affected by the heritability of selfing. Therefore, the correlation in homozygosity across all loci of an individual needs to be taken into account to achieve an unbiased estimate of selfing rate based on descent measures. We can quantify the bias of estimates of selfing rate that are based on the traditional population structure approach. Equation 1 reduces to the familiar $F=S /(2-S)$ when the selfing rate of parents has no effect on the selfing rate of offspring $(\rho=S)$. The derivation of this classic result assumes that each individual is equally likely to self, regardless of its ancestry. However, if $\rho \neq S$ then the population structure estimate of selfing rate is biased and overestimates the real selfing rate of populations by as much as a factor of two when $S$ is small. This is shown by the plot of relative bias in Figure 1, which plots the relative difference between the population structure estimate of selfing rate, $\hat{S}=2 F /(1+F)$ and its true value $S$, as a function of $h^{2}$. The mathematical expression for Figure 1 uses Equation 5 with $\rho$ substituted out by the relation from Equation 7 to yield $\left(h^{2}-h^{2} S\right) /\left(2+h^{2} S-h^{2}\right)$. Figure 1 shows that estimates of selfing rate from populations with high heritability and/or low to intermediate selfing rates are likely to overestimate, sometimes substantially, the real selfing rate of the population.

\section{Likelihood model}

To be able to estimate selfing rate more accurately and to find estimates of the heritability of selfing rate from empirical data, information from all loci should be used together. Therefore, we propose a likelihood model that is based on the probability of an individual having a particular multilocus genotype. To find the log-likelihood of each individual's genotype, we make two simplifying assumptions: that there is no mutation and that segregation happens independently at each locus. (As a result of the latter assumption, the method should be applied to loci that show no evidence of linkage.) That is to say, given a pattern of selfing and outcrossing within the specific line of ancestry that leads to a particular individual, we will use that history of selfing to calculate the probability of identity of the alleles at each locus independently. We use $h^{2}$ substituted for $\rho$ in $\varphi(x, S, \rho)$ (according to Equation 7) so that

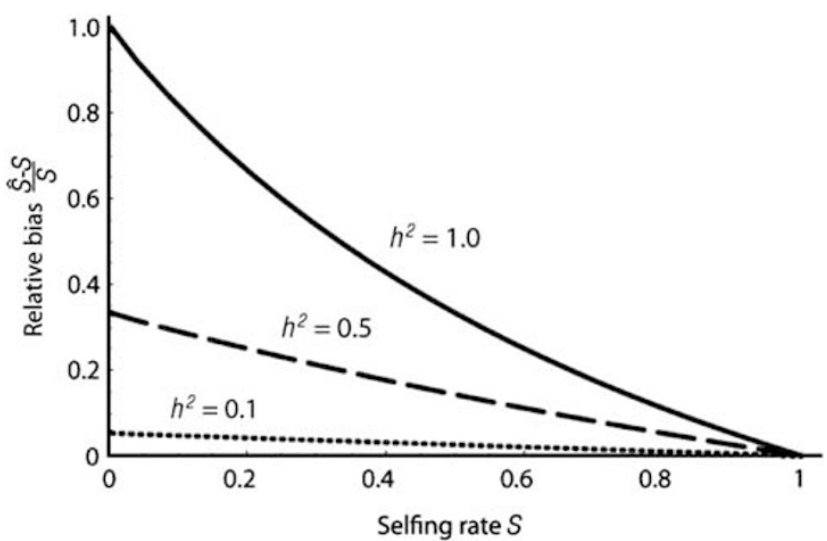

Figure 1 The relative bias of the population structure estimate of selfing rate $(\hat{S}=2 F /(1+F))$ as a function of the population's true selfing rate $S$ and its heritability $h^{2}$, yielding the expression $\left(h^{2}-h^{2} S\right) /\left(2+h^{2} S-h^{2}\right)$. $\varphi\left(x, S, h^{2}\right)$ describes the distribution of ancestral selfing generations $(x)$ in a lineage since the last ancestor that outcrossed. Equation 1 gives the inbreeding coefficient $F(x)$ for a given $x$. The probability of an individual having a particular genotype is the product across all loci of the probability of that individual's genotype per locus for a given $x$, times the probability of that $x$, added over all $x$.

$$
\begin{aligned}
L\left[\text { individual } j \mid S, h^{2}\right]= & \sum_{x=0}^{\infty} \varphi\left(x, S, h^{2}\right) \\
& \times \prod_{\substack{l \in \text { all heterozygous } \\
\text { loci }}}\left(2 p_{\mathrm{li}} p_{\mathrm{lk}}(1-F(x))\right) \\
& \times \prod_{\substack{l \in \text { all homozygous } \\
\text { loci }}} p_{\mathrm{li}}^{2}(1-F(x))+p_{\mathrm{li}} F(x)
\end{aligned}
$$

where $p_{\mathrm{li}}$ is the allele frequency of allele $\mathrm{i}$ at locus 1 .

Equation 8 gives the likelihood of the whole genotype for individual $j$, given the parameters $\left\{S, h^{2}\right\}$. We now wish to incorporate a measure of scoring error identical to the misscoring by David et al. (2007). We use the error term $E_{1}$ to denote the probability that a heterozygote is erroneously scored as a homozygote at locus 1 . Thus, the likelihood of the whole apparent genotype of individual $j$ given the parameters $\left\{S, h^{2}, E\right\}$ with the vector $E=\left(E_{1}, E_{2}, \ldots\right.$, $\left.E_{1}\right)$ of error terms is:

$$
\begin{aligned}
L\left[\text { individual } j \mid S, h^{2}, E\right]= & \sum_{x=0}^{\infty} \varphi\left(x, S, h^{2}\right) \\
& \times \prod_{\substack{l \in \text { all apparently } \\
\text { heterozygous loci }}}\left[2 p_{\mathrm{li}} p_{\mathrm{lk}}(1-F(x))\left(1-E_{l}\right)\right] \\
& \times \prod_{\substack{l \in \text { all apparently } \\
\text { homozygous loci }}}\left[\begin{array}{c}
p_{\mathrm{li}}^{2}(1-F(x))+p_{\mathrm{li}} F(x) \\
+p_{\mathrm{li}}\left(1-p_{\mathrm{li}}\right)(1-F(x)) E_{l}
\end{array}\right] .
\end{aligned}
$$

We will also consider a likelihood model when the rate of scoring error is constant but unknown $\left(E_{1}=E\right)$ for all loci:

$$
\begin{aligned}
L\left[\text { individual } j \mid S, h^{2}, E\right]= & \sum_{x=0}^{\infty} \varphi\left(x, S, h^{2}\right) \\
& \times \prod_{\substack{l \in \text { all apparently } \\
\text { heterozygous loci }}}\left[2 p_{\mathrm{li}} p_{\mathrm{lk}}(1-F(x))(1-E)\right] \\
& \times \prod_{\substack{l \in \text { all apparently } \\
\text { homozygous loci }}}\left[\begin{array}{l}
p_{\mathrm{li}}^{2}(1-F(x))+p_{\mathrm{li}} F(x) \\
+p_{\mathrm{li}}\left(1-p_{\mathrm{li}}\right)(1-F(x)) E
\end{array}\right] .
\end{aligned}
$$

Taking the log of the likelihood equation and summing over all individuals in a sample gives the log-likelihood for the parameter set. If the result is multiplied by minus one, we get the negative log-likelihood:

$$
L=-\sum_{j=1}^{N} \log \left[L_{j}\right]
$$

The full set of parameters in the log-likelihood function would technically include estimates of allele frequencies as nuisance parameters. Furthermore, we only consider a single kind of scoring error that is when heterozygotes are erroneously scored as homozygotes at a locus. It is not our intent to evaluate scoring artifacts so much as we include a measure of scoring error as a means to robustly estimate $S$ and $h^{2}$.

To calculate maximum-likelihood estimates for selfing rate and heritability of selfing rate, we created a tailored maximum-likelihood estimation function that uses the nlminb optimizer in the $\mathrm{R}$ computing environment (R Development Core Team, 2010). The summation in Equations 9 and 10 are evaluated for $x=0$ to $x=1000$ ancestral selfing generations because it was found that higher values of $x$ were needed for accuracy in simulated populations with high $S$ and $h^{2}$. We also implemented the likelihood function (with an nlminb optimizer) using mle2 (Bolker and R Development Core Team, 2011), which provides added functionality allowing the user to calculate confidence intervals for estimates. $\mathrm{R}$ code for both procedures is available in the Supplementary information. The results presented in the paper used the 
first procedure. In addition, all MESH methods provided in the Supplementary information are for bi-allelic data.

To show how estimates from MESH is compared with other methods of estimating selfing rate in the presence of scoring artifacts, we also estimated selfing rate using a maximum-likelihood estimation method based on the likelihood equation published in David et al. (2007). Although this method also makes use of multilocus correlations in homozygosity resulting from multiple generations of selfing, it does not account for an intergenerational correlation in selfing rate that increases the probability of multihomozygous genotypes. We used the approximation for homozygous loci with more than 20 generations of selfing as suggested by David et al. (2007).

\section{Simulation methods}

In order to test the performance of the likelihood method, we simulated populations with various selfing rates and heritabilities. Simulations were done in the statistical package R ( $\mathrm{R}$ Development Core Team, 2010). The data analyzed here are specific to a bi-allelic scenario; however, the general principles used in MESH should extend to multiallelic data sets. All simulated conditions were done using specified values of $S$ and $\rho, 10$ or 100 loci, 500 generations, an initial allele frequency of 0.5 and a population size of 1000 . We analyzed the population data in the 500th generation, well after the parameters equilibrated at a maximum of approximately 50 generations. The 0th (parental) generation used a uniform distribution random number generator and the starting allele frequencies to create the genotype of each individual. All individuals of the starting generation were produced by outcrossing. To simulate a reproductive episode, an individual is randomly chosen from the population (parental generation) and its selfing history is used to determine the probability that the individual reproduces by outcrossing or reproduces by selfing. For a specified parameter set $\{S, \rho\}$, the probability that a parent reproduces by selfing is equal to $\rho$ if the parent was itself produced by selfing (that is, grandparent selfed). Alternatively, if the grandparent outcrossed, then the probability that the parent selfs is equal to $S(1-\rho) /(1-S)$, which maintains the population selfing rate at $S$. The complete transition probability matrix is therefore:

$\begin{array}{ccc} & \text { Parent selfs } & \text { Parent outcrosses } \\ \text { Grandparent selfed } & \rho & 1-\rho \\ \text { Grandparent outcrossed } & S(1-\rho) /(1-S) & (S(\rho-2)+1) /(1-S)\end{array}$

If an individual reproduces by outcrossing, then a pollen parent (male gamete donor) is chosen from the population at random. Each reproductive episode is an independent event, and new individuals are created in this way until the population size of the offspring generation equals the population size of the parental generation.

Scoring artifacts were also simulated to provide imperfect population genetic data. The rate of scoring error was set for each locus $\left(E_{1}\right)$ and heterozygous genotypes were randomly scored incorrectly as homozygous by changing the first allele of the locus to the other allele type. All simulations used an $E=(0,0.01,0.05,0.1,0.01,0.05,0.1,0,0.1,0.05)$ vector of scoring error for every 10 loci, such that the average scoring error was $4.7 \%$.

\section{RESULTS}

We use the multilocus estimation of selfing rate and its heritability (MESH) method given by Equation 10 to evaluate estimates of selfing rate and heritability of selfing rate from simulated data in the presence of scoring error. The results from Tables 1-3 show that this method can improve estimates of selfing rate while also providing an estimate of heritability of selfing rate. We will highlight some of the important differences between estimates of $S$ and $h^{2}$ as well as the effect of increasing the number of individuals and the number of loci of sampled individuals in a data set. We will also compare estimates of $S$ to estimates from a maximum-likelihood estimation method based on the likelihood equation from David et al. (2007). Lastly, we will evaluate estimates of $S$ and $h^{2}$ from the fully parameterized MESH model (Equation 9).

Table 1 shows that the estimated bias of $S$ and $h^{2}$ estimates is low in absolute terms, but can be proportionally large when the true heritability is small. Estimates of $S$ are slightly negatively biased when $h^{2}$ is low and positively biased when $h^{2}$ is high, whereas the estimated bias of $h^{2}$ is greater in magnitude and usually of the opposite sign. Estimates of $S$ also have a lower root mean squared error than $h^{2}$ estimates. The root mean squared error of $h^{2}$ estimates generally improves as the parametric value of $h^{2}$ increases with the smallest root mean squared error occurring for intermediate $S$.

For every parametric combination of $S$ and $h^{2}$ except with $h^{2}=0$ or the combination $S=0.9$ and $h^{2}=0.2$, MESH estimates of $S$ were less biased and had lower root mean squared error than estimates from a maximum-likelihood estimation method based on the likelihood equation from David et al. (2007) (see Table 1).

Table 1 The bias and RMSE of $S, h^{2}$ and $E$ estimates from MESH

\begin{tabular}{|c|c|c|c|c|c|c|c|c|c|c|}
\hline \multicolumn{2}{|c|}{ Simulated conditions } & \multirow{2}{*}{$\begin{array}{c}\mathrm{F} \\
\operatorname{Bias}(\hat{\mathrm{S}})\end{array}$} & \multicolumn{6}{|c|}{ MESH } & \multicolumn{2}{|c|}{ Likelihood equation from David et al. (2007) } \\
\hline S & $h^{2}$ & & $\operatorname{Bias}(\hat{\mathrm{S}})$ & $R M S E(\hat{S})$ & $\operatorname{Bias}\left(\hat{\mathrm{h}}^{2}\right)$ & $R M S E\left(\hat{\mathrm{h}}^{2}\right)$ & $\operatorname{Bias}(\hat{E})$ & $R M S E(\hat{E})$ & $\operatorname{Bias}(\hat{\mathrm{S}})$ & $R M S E(\hat{S})$ \\
\hline 0.1 & 0 & 0 & $-0.0132^{*}$ & 0.0308 & $0.1256^{*}$ & 0.2221 & $0.0038 *$ & 0.0173 & 0.0008 & 0.0280 \\
\hline 0.1 & 0.2 & 0.0099 & -0.0007 & 0.0321 & $0.0301^{*}$ & 0.2157 & -0.0008 & 0.0180 & $0.0293^{*}$ & 0.0404 \\
\hline 0.1 & 0.5 & 0.0290 & $0.0024 *$ & 0.0291 & -0.0075 & 0.1778 & $-0.0027^{*}$ & 0.0178 & $0.0829 *$ & 0.0881 \\
\hline 0.1 & 0.8 & 0.0563 & $0.0030 *$ & 0.0281 & $-0.0287^{*}$ & 0.1577 & $-0.0027^{*}$ & 0.0176 & $0.1509 *$ & 0.1563 \\
\hline 0.1 & 0.9 & 0.0681 & $0.0105^{*}$ & 0.0423 & $-0.0770 *$ & 0.2348 & $-0.0052^{*}$ & 0.0239 & $0.1806^{*}$ & 0.1905 \\
\hline 0.5 & 0 & 0 & $-0.0176^{*}$ & 0.0352 & $0.0652^{*}$ & 0.1129 & $0.0082^{*}$ & 0.0273 & -0.0003 & 0.0221 \\
\hline 0.5 & 0.2 & 0.0263 & -0.0021 & 0.0370 & 0.0052 & 0.1210 & -0.0010 & 0.0285 & $0.0534 *$ & 0.0573 \\
\hline 0.5 & 0.5 & 0.0714 & 0.0001 & 0.0349 & -0.0010 & 0.0994 & $-0.0019^{*}$ & 0.0272 & $0.1361^{*}$ & 0.1376 \\
\hline 0.5 & 0.8 & 0.1250 & 0.0003 & 0.0374 & $-0.0053^{*}$ & 0.0745 & $-0.0018^{*}$ & 0.0280 & $0.2203^{*}$ & 0.2215 \\
\hline 0.5 & 0.9 & 0.1452 & $0.0049^{*}$ & 0.0443 & $-0.0094^{*}$ & 0.0625 & $-0.0044^{*}$ & 0.0292 & $0.2502^{*}$ & 0.2518 \\
\hline 0.9 & 0 & 0 & $-0.0114^{*}$ & 0.0215 & $0.1200^{*}$ & 0.2042 & $0.0236^{*}$ & 0.0638 & 0.0001 & 0.0097 \\
\hline 0.9 & 0.2 & 0.0091 & $-0.0056^{*}$ & 0.0209 & $0.0441^{*}$ & 0.1962 & $0.0102 *$ & 0.0611 & $0.0157 *$ & 0.0179 \\
\hline 0.9 & 0.5 & 0.0231 & $-0.0016^{*}$ & 0.0188 & -0.0018 & 0.1715 & 0.0008 & 0.0559 & $0.0370 *$ & 0.0377 \\
\hline 0.9 & 0.8 & 0.0375 & $0.0017^{*}$ & 0.0200 & $-0.0143^{*}$ & 0.093 & $-0.0094^{*}$ & 0.0465 & $0.0573 *$ & 0.0578 \\
\hline 0.9 & 0.9 & 0.0424 & $0.0062^{*}$ & 0.0235 & $-0.0336^{*}$ & 0.0632 & $-0.0225^{*}$ & 0.0421 & $0.0635^{*}$ & 0.0709 \\
\hline
\end{tabular}

Abbreviations: $\mathrm{MESH}$, multilocus estimation of selfing and its heritability; RMSE, root mean squared error.

Statistics are based on 1000 replicates of simulated bi-allelic data with specified $S$ and $h^{2}, 1000$ individuals, 10 loci and 500 generations. A vector $E=(0,0.01,0.05,0.1,0.01,0.05,0.1,0$,

$0.1,0.05$ ) was used to set a variable scoring error among loci. For comparison we include the predicted bias of $S$ estimates from the inbreeding coefficient $F$ as well as statistics for estimates of $S$ based on the likelihood equation from David et al. (2007). An asterisk indicates that the estimated bias is significantly different from 0 (based on bootstrap $95 \%$ confidence intervals). 
Table 2 The bias and RMSE of $S$ estimates from MESH for varying number of loci and number of individuals in a sample

\begin{tabular}{|c|c|c|c|c|}
\hline \multirow[t]{2}{*}{ Number of individuals (N) } & \multicolumn{4}{|c|}{ Number of loci $\left(\mathrm{n}_{l}\right)$} \\
\hline & 10 & 20 & 30 & 100 \\
\hline \multicolumn{5}{|l|}{ Bias } \\
\hline 10 & -0.0114 & -0.0141 & 0.0008 & 0.0001 \\
\hline 30 & -0.0076 & -0.0022 & 0.0018 & -0.0018 \\
\hline 100 & -0.0064 & 0.0063 & 0.0042 & 0.0018 \\
\hline 300 & -0.0017 & 0.0031 & 0.0029 & -0.0003 \\
\hline 600 & 0.0023 & 0.0031 & 0.0009 & 0.0002 \\
\hline 1000 & 0.0027 & 0.0015 & 0.0010 & 0.0006 \\
\hline \multicolumn{5}{|l|}{ RMSE } \\
\hline 10 & 0.1147 & 0.1034 & 0.1073 & 0.1009 \\
\hline 30 & 0.0912 & 0.0723 & 0.0670 & 0.0619 \\
\hline 100 & 0.0644 & 0.0485 & 0.0409 & 0.0324 \\
\hline 300 & 0.0460 & 0.0300 & 0.0242 & 0.0185 \\
\hline 600 & 0.0365 & 0.0196 & 0.0159 & 0.0125 \\
\hline 1000 & 0.0284 & 0.0158 & 0.0135 & 0.0107 \\
\hline
\end{tabular}

Abbreviation: RMSE, root mean squared error

Statistics are based on 100 replicates of sampled bi-allelic data with $n_{1}$ loci and $N$ number of individuals from which the original data set was simulated with $S=0.1, h^{2}=0.5,1000$ individuals, 100 loci and 500 generations. A vector $E=(0,0.01,0.05,0.1,0.01,0.05,0.1$ $0,0.1,0.05)$ per 10 loci was used to set a variable scoring error among loci after sampling. $0,0.1,0.05)$ per 10 loci was used to set a variable scoring error among loci after samp
None of the estimated bias is significantly different from 0 (based on bootstrap $95 \%$ None of the estimated
confidence intervals).

Table 3 The bias and RMSE of $h^{2}$ estimates from MESH for varying number of loci and number of individuals in a sample

\begin{tabular}{|c|c|c|c|c|}
\hline \multirow[t]{2}{*}{ Number of individuals (N) } & \multicolumn{4}{|c|}{ Number of loci $\left(\mathrm{n}_{p}\right)$} \\
\hline & 10 & 20 & 30 & 100 \\
\hline \multicolumn{5}{|l|}{ Bias } \\
\hline 10 & $-0.1531^{*}$ & $-0.2502^{*}$ & $-0.2597^{*}$ & $-0.1307^{*}$ \\
\hline 30 & -0.0381 & $-0.0981^{*}$ & $-0.1244^{*}$ & $-0.0925^{*}$ \\
\hline 100 & 0.0238 & $-0.0649^{*}$ & $-0.0567^{*}$ & $-0.0527^{*}$ \\
\hline 300 & -0.0110 & $-0.0435^{*}$ & $-0.0419^{*}$ & $-0.0320^{*}$ \\
\hline 600 & -0.0149 & $-0.0363^{*}$ & $-0.0205^{*}$ & $-0.0146^{*}$ \\
\hline 1000 & -0.0217 & -0.0135 & -0.0074 & -0.0051 \\
\hline \multicolumn{5}{|l|}{ RMSE } \\
\hline 10 & 0.4795 & 0.4386 & 0.4006 & 0.2800 \\
\hline 30 & 0.4461 & 0.3816 & 0.3542 & 0.2661 \\
\hline 100 & 0.3843 & 0.2686 & 0.2228 & 0.1587 \\
\hline 300 & 0.2835 & 0.1482 & 0.1215 & 0.0908 \\
\hline 600 & 0.2325 & 0.1120 & 0.0841 & 0.0620 \\
\hline 1000 & 0.1687 & 0.0823 & 0.0684 & 0.0516 \\
\hline
\end{tabular}

Abbreviations: MESH, multilocus estimation of selfing and its heritability; RMSE, root mean squared error.

Statistics are based on 100 replicates of sampled bi-allelic data with $n_{\downarrow}$ loci and $N$ number of individuals from which the original data set was simulated with $S=0.1, h^{2}=0.5,1000$

individuals, 100 loci and 500 generations. A vector $E=(0,0.01,0.05,0.1,0.01,0.05,0.1$

$0,0.1,0.05)$ per 10 loci was used to set variable scoring error among loci after sampling. An asterisk indicates that the estimated bias is significantly different from 0 (based on bootstrap $95 \%$ confidence intervals).

Tables 2 and 3 demonstrate the effect of increasing number of loci and number of individuals in a sample on the bias and root mean squared error of $S$ and $h^{2}$ estimates. The root mean squared error of $S$ and $h^{2}$ decreases as the multiple of number of loci and number of individuals $\left(n_{1} \times N\right)$ increases; however, increasing the number of individuals generally improves the estimates (reduces the root mean
Table 4 The bias and RMSE of $S$ and $h^{2}$ estimates from the fully parameterized MESH method (Equation 9) that estimates a scoring error for each locus $\left(E_{l}\right)$

\begin{tabular}{|c|c|c|c|c|c|}
\hline \multicolumn{2}{|c|}{ Simulated conditions } & \multicolumn{4}{|c|}{ MESH } \\
\hline S & $h^{2}$ & $\operatorname{Bias}(\hat{\mathrm{S}})$ & $R M S E(\hat{S})$ & $\operatorname{Bias}\left(\hat{\mathrm{h}}^{2}\right)$ & $R M S E\left(\hat{\mathrm{h}}^{2}\right)$ \\
\hline 0.1 & 0 & $-0.0235^{*}$ & 0.0342 & $0.1754 *$ & 0.2649 \\
\hline 0.1 & 0.2 & $-0.0129 *$ & 0.0297 & $0.0869 *$ & 0.2203 \\
\hline 0.1 & 0.5 & $-0.0095^{*}$ & 0.0254 & $0.0490 *$ & 0.1623 \\
\hline 0.1 & 0.8 & $-0.0067^{*}$ & 0.0241 & $0.0188 *$ & 0.1275 \\
\hline 0.1 & 0.9 & $-0.0042^{*}$ & 0.0303 & 0.0007 & 0.1446 \\
\hline 0.5 & 0 & $-0.0303^{*}$ & 0.0419 & $0.0973^{*}$ & 0.1388 \\
\hline 0.9 & 0 & $-0.0212^{*}$ & 0.0282 & $0.2060 *$ & 0.2703 \\
\hline 0.9 & 0.2 & $-0.0169 *$ & 0.0258 & $0.1470^{*}$ & 0.2295 \\
\hline
\end{tabular}

Abbreviations: MESH, multilocus estimation of selfing and its heritability; RMSE, root mean squared error.

Statistics are based on 1000 replicates of simulated bi-allelic data with specified $S$ and $h^{2}$, 1000 individuals, 10 loci and 500 generations. The results for $h^{2}=0$ and the combination $S=0.9$ and $h^{2}=0.2$ each used 1000 replicates otherwise 500 replicates were used. A vector $E=(0,0.01,0.05,0.1,0.01,0.05,0.1,0,0.1,0.05)$ was used to simulate variable scoring error among loci. An asterisk indicates that the estimated bias is significantly different from 0 (based on bootstrap 95\% confidence intervals).

squared error) more than increasing the number of loci for the same total genotyping effort $\left(n_{1} \times N\right)$. The estimated bias of $S$ and $h^{2}$ estimates also decreases as the number of individuals in a sample increase. Although increasing the number of loci generally reduces the bias of $S$ estimates, its effect on the bias of $h^{2}$ estimates is not clear.

Estimates of $S$ and $h^{2}$ from the fully parameterized MESH model are provided for selected parametric combinations of $S$ and $h^{2}$ in the presence of scoring error (see Table 4). The computing time required to obtain estimates for all parameters is substantially longer while the estimates of $S$ and $h^{2}$ are generally less well behaved-having larger root mean squared error and bias-than estimates from the MESH method with a single parameter for scoring error. MESH (fully parameterized and single scoring error parameter methods) was also tested using allozyme data for Physa acuta (from David et al., 2007). These results are available in the Supplementary information.

\section{DISCUSSION}

We have presented a new method to estimate selfing rate $(S)$ and heritability of selfing rate $\left(h^{2}\right)$ in mixed mating populations using genotype frequency data from a single generation. We have demonstrated that the correlation in selfing rate between generations can bias estimates of the population's selfing rate. By accounting for the probability that parents of selfers also reproduced by selfing $(\rho)$, we can correct this bias and also estimate the heritability of selfing rate.

Our method follows from the idea that in a mixed mating population a lineage's change in heterozygosity may depend on its history of selfing. Correlation of homozygosity among loci is an evidence of a correlation in selfing rate between generations. We have shown that selfing rate estimates based on the conventional population structure method likely overestimate the true selfing rate of the population by as much as a factor of two. This suggests that some previous estimates of selfing rate that used the population structure method will need to be updated to correct for this bias.

We have devised a method of estimating selfing rate and heritability of selfing rate based upon a maximum-likelihood approach that uses data from multilocus genotypes of sampled individuals. Similar to other methods based on a population structure approach, the MESH method assumes that the mixed mating population is at inbreeding equilibrium. Therefore, even though we suggest that there may exist a 
possible intergenerational correlation in selfing rate, the population selfing rate is assumed to not to change over generations. The MESH method is robust to scoring errors and shows improved results when compared with other estimates of selfing rate. The methodology of MESH differs from robust multilocus estimation of selfing rate (see David et al., 2007) in several ways. Most importantly, MESH estimates selfing rate as well as its heritability by accounting for the intergenerational correlation in selfing rate within lineages.

We found that estimates of selfing rate from MESH were generally more accurate and precise than estimates from a maximum-likelihood estimation method based on the likelihood equation from David et al. (2007). In particular, as the parametric value of $h^{2}$ increased, there was a steady increase in bias and root mean squared error of $S$ estimates from a maximum-likelihood estimation method based on the likelihood equation from David et al. (2007). This suggests that estimates of $S$ from this method are biased by the intergenerational correlation in selfing rate as predicted in Figure 1. It is not surprising then that when the parametric value of $h^{2}=0$, estimates of $S$ from a maximum-likelihood estimation method based on the likelihood equation from David et al. (2007) were less biased and had a lower mean root squared error than MESH estimates of $S$. This is because the likelihood equation from David et al. (2007) implicitly assumes that heritability of selfing rate is 0 , whereas MESH estimates of $S$ are subject to error in estimating $h^{2}$. However, it is not reasonable to assume without evidence that the heritability of selfing rate is 0 in natural populations. In general, estimates of $S$ from MESH were usually better than estimates from the maximum-likelihood estimation method from David et al. (2007).

The fully parameterized MESH method did not perform as well as the MESH method with a single scoring error parameter. The reason for this is in part due to the fact that there is likely not sufficient information in the simulated data to estimate individual scoring errors, and as a result uncertainty in estimates of scoring error for each locus will affect the accuracy and precision of estimates of $S$ and $h^{2}$. Therefore, we recommend using MESH with a single parameter scoring error when there is an a priori expectation that scoring error occurs at a low rate; however, this method should not be used when there is extreme heterogeneity in scoring error among loci, in which case the fully parameterized method may be better suited. We also recommend that any locus that is known to have a high error rate should not be included in a MESH analysis.

Our simulations predict the kinds of sample sizes that may be required for reasonable precision and accuracy of estimates. Increasing the number of individuals in a sample set and increasing the number of loci of sampled individuals reduced the root mean squared error among estimates of $S$ and $h^{2}$. However, increasing the number of individuals reduces root mean squared error more than increasing the number of loci for the same multiple of individuals and loci. This suggests that information on the selfing history of each genotyped individual is maximized quickly for increasing number of loci (there is little difference in estimates from 20 to 30 to 100 loci), while more individuals provide added information about the population parameters $S$ and $h^{2}$. Increasing the number of individuals reduces the estimated bias of the parameters, whereas increasing the number of loci did not have a clear effect. For a researcher, these findings demonstrate the need for a sample that includes as many individuals as possible and a large number of loci. The MESH method requires larger sample sizes than other methods that only estimate selfing, because heritability, being a second-order parameter, requires more information for a reliable estimate. Researchers who wish to use this method are advised to collect sample sizes based on the information in Tables 2 and 3, depending on the desired precision.
In addition to the challenges of getting a large enough sample size, this method of estimating the heritability of selfing rate has other constraints. We have assumed that loci segregate independently and that there is no mutation, requiring that the marker loci be chosen such that they show no strong linkage disequilibrium. In addition, the method does not consider the effect of bi-parental inbreeding on probabilities of identity. This is an important factor since bi-parental inbreeding also increases homozygosity of loci, which may cause overestimation of selfing rate in predominantly outcrossing populations (David et al., 2007). Furthermore, the estimate of heritability is based on the correlation in nature between parents and offspring, and this correlation can be affected by genetic similarities, shared environments and maternal effects. Thus the estimates we derive should be viewed as an upper-bound for the heritability, because environmental associations between parents and offspring are likely to be present in a natural setting. In addition, to arrive at an estimate of heritability we have reasoned that an intergenerational correlation in selfing rate will result from those individuals in a selfing lineage being at least as likely to self than predicted by the average selfing rate of the population. However, we have not considered the situation in which those individuals arising from a selfing lineage may be more prone to outcross than predicted by the average selfing rate, perhaps as a mechanism to avoid further inbreeding depression. Such an idea should be further explored before we can conceive how this might affect selfing rate estimates. More research is therefore needed before we will know how improving methods of estimation may affect the distribution of selfing rates reported in Schemske and Lande (1985) and Goodwillie et al. (2005).

The results indicate that the MESH method provides precise and accurate estimates of selfing rate, whereas estimates of heritability of selfing rate are more qualitative. These findings suggest that by using this method a researcher can be confident that they will obtain a quantitative estimate of the population's true selfing rate that accounts for the intergenerational correlation in selfing rate. Estimates of heritability of selfing rate, while not as precise, may be used as a qualitative descriptor, providing the researcher with a method of distinguishing low, intermediate and high heritability.

\section{DATA ARCHIVING}

Data sets have been deposited in the Dryad repository: doi:10.5061/ dryad.nf2c425n.

Supplementary information is available for this article and includes analysis of data from Physa acuta, estimates of scoring error from a fully parameterized model (Equation 9) and the R code for MESH.

\section{CONFLICT OF INTEREST}

The authors declare no conflict of interest.

\section{ACKNOWLEDGEMENTS}

This work was funded by a Discovery grant and an Undergraduate Student Research Award from the Natural Science and Engineering Research Council (Canada). We are grateful to Mark Johnston and anonymous reviewers for comments on this manuscript and to Patrice David for sharing data on Physa acuta.

Avise JC (2011). Hermaphroditism: A Primer on the Biology, Ecology, and Evolution of Dual Sexuality. Columbia University Press: New York.

Barrett SC (2002). The evolution of plant sexual diversity. Nat Rev Genet 3: 274-284.

Bolker B, R Development Core Team (2011). bbmle: Tools for general maximum likelihood estimation. $\mathrm{R}$ package version 1.0.3. http://CRAN.R-project.org/package=bbmle.

Charlesworth D, Willis JH (2009). The genetics of inbreeding depression. Nat Rev Genet 10: 783-796. 
Cockerham CC, Weir BS (1973). Descent measures for two loci with some applications. Theor Popul Biol 4: 300-330.

David P, Pujol B, Viard F, Castella V, Goudet J (2007). Reliable selfing rate estimates from imperfect population genetic data. Mol Ecol 16: 2474-2487.

Escobar JS, Auld JR, Correa AC, Alonso JM, Bony YK, Coutellec M et al. (2011). Patterns of mating-system evolution in hermaphroditic animals: correlations among selfing rate, inbreeding depression, and the timing of reproduction. Evolution 65: 1233-1253.

Fyfe JL, Bailey NTJ (1951). Plant breeding studies in leguminous forage crops. I. Natural cross-breeding in winter beans. J Agric Sci 41: 371-378.

Goodwillie C, Kalisz S, Eckert CG (2005). The evolutionary enigma of mixed mating systems in plants: Occurrence, theoretical explanations, and empirical evidence. Annu Rev Ecol Evol Syst 36: 47-79.
Jarne P, Auld JR (2006). Animals mix it up too: the distribution of self-fertilization among hermaphroditic animals. Evolution 60: 1816-1824.

Jarne P, David P (2008). Quantifying inbreeding in natural populations of hermaphroditic organisms. Heredity 100: 431-439.

Lande R, Schemske DW (1985). The evolution of self-fertilization and inbreeding depression in plants. I. genetic models. Evolution 39: 24-40.

R Development Core Team (2010). R: A LANGUAGE and Environment for Statistical Computing. R Foundation for Statistical Computing: Vienna, Austria, ISBN 3-90005107-0, URL. http://www.R-project.org.

Ritland K, Jain SK (1981). A model for the estimation of outcrossing rate and gene frequencies using $n$ independent loci. Heredity 47: 35-52.

Schemske DW, Lande R (1985). The evolution of self-fertilization and inbreeding depression in plants. II. Empirical observations. Evolution 39: 41-52.

Supplementary Information accompanies the paper on Heredity website (http://www.nature.com/hdy) 